\title{
Statistically Preserved Structures and Anomalous Scaling in Turbulent Active Scalar Advection
}

\author{
Emily S.C. Ching \\ Department of Physics, The Chinese University of Hong Kong, Sha Tin, Hong Kong \\ Yoram Cohen, Thomas Gilbert, and Itamar Procaccia \\ Dept. of Chemical Physics, The Weizmann Institute of Science, Rehovot 76100, Israel
}

(Dated: February 9, 2020)

\begin{abstract}
The anomalous scaling of correlation functions in the turbulent statistics of active scalars (like temperature in turbulent convection) is understood in terms of an auxiliary passive scalar which is advected by the same turbulent velocity field. While the odd-order correlation functions of the active and passive fields differ, we propose that the even-order correlation functions are the same to leading order (up to a trivial multiplicative factor). The leading correlation functions are statistically preserved structures of the passive scalar decaying problem, and therefore universality of the scaling exponents of the even-order correlations of the active scalar is demonstrated.
\end{abstract}

The old riddle of anomalous scaling in generic turbulent statistics had been solved recently, albeit in the modest context of passive scalar and passive vector advection [1, 2, 3]. Motivated by some recent numerical indications 㭡, we propose in this Letter that under generic conditions a similar understanding can be extended to active scalar (or vector) advection by a generic turbulent velocity field. The difference between the two problems is that passive fields leave the velocity statistics intact, satisfying a purely linear problem into which the velocity field is injected as an advection term, while active fields influence the statistics of the velocity field. This development brings us therefore closer to understanding anomalous scaling in the nonlinear velocity problem itself.

Passive fields are advected in turbulence with typical dynamics of the form

$$
\frac{\partial \phi(\boldsymbol{r}, t)}{\partial t}+\boldsymbol{u}(\boldsymbol{r}, t) \cdot \boldsymbol{\nabla} \phi(\boldsymbol{r}, t)=\kappa \nabla^{2} \phi(\boldsymbol{r}, t)+f(\boldsymbol{r}, t) .
$$

Here the field $\phi(\boldsymbol{r}, t)$ can be a passive scalar $c(\boldsymbol{r}, t)$ or passive vector $\boldsymbol{v}(\boldsymbol{r}, t) . \kappa$ is the diffusivity and $f(\boldsymbol{r}, t)$ is a white random force of zero mean with compact support in $\boldsymbol{k}$-space, acting on the largest scales of the order of the outer scale $L$ only. The advecting velocity field is a generic turbulent field at high Reynolds number with an extended scaling range, typically exhibiting anomalous scaling. We use the word "generic" here to distinguish from model velocity fields with $\delta$-function temporal correlations [5]. "Anomalous scaling" means that multi-point correlation functions are homogeneous functions of their arguments, with exponents that cannot be guessed from dimensional analysis. Thus for example the field $\phi(\boldsymbol{r}, t)$ has simultaneous multi-point correlation functions

$$
F^{(n)}\left(\boldsymbol{r}_{1}, \boldsymbol{r}_{2}, \cdots, \boldsymbol{r}_{n}\right) \equiv\left\langle\phi\left(\boldsymbol{r}_{1}, t\right) \phi\left(\boldsymbol{r}_{2}, t\right) \cdots \phi\left(\boldsymbol{r}_{n}, t\right)\right\rangle_{f},
$$

where pointed brackets with subscript $f$ refer to averaging over the statistics of the advecting velocity field and of the forcing. Anomalous scaling means that

$$
F^{(n)}\left(\lambda \boldsymbol{r}_{1}, \cdots, \lambda \boldsymbol{r}_{n}\right)=\lambda^{\zeta_{n}} F^{(n)}\left(\boldsymbol{r}_{1}, \cdots, \boldsymbol{r}_{n}\right),
$$

with $\zeta_{n}$ having a non-trivial dependence on $n$.

In recent work [2, 3] it was clarified why and how passive fields exhibit anomalous scaling. The key is to consider a problem associated with Eq. (11) which is the decaying problem in which the forcing $f(\boldsymbol{r}, t)$ is put to zero. The problem becomes then a linear initial value problem, $\frac{\partial \phi}{\partial t}=\mathcal{L} \phi$, with a formal solution

$$
\phi(\boldsymbol{r}, t)=\int d \boldsymbol{r}^{\prime} \boldsymbol{R}\left(\boldsymbol{r}, \boldsymbol{r}^{\prime}, t\right) \phi\left(\boldsymbol{r}^{\prime}, 0\right),
$$

with the operator $\boldsymbol{R} \equiv T^{+} \exp \left(\int_{0}^{t} d s \mathcal{L}(s)\right)$, and $T^{+}$being the time ordering operator. Define next the now time dependent correlation functions of the decaying problem:

$$
C^{(n)}\left(\boldsymbol{r}_{1}, \cdots, \boldsymbol{r}_{n}, t\right) \equiv\left\langle\phi\left(\boldsymbol{r}_{1}, t\right) \cdots \phi\left(\boldsymbol{r}_{n}, t\right)\right\rangle .
$$

Pointed brackets without subscript $f$ refer to the decaying object in which averaging is with respect to realizations of the velocity field only. As a result of Eq. (4) the decaying correlation functions are developed by a propagator $\mathcal{P}_{\underline{\boldsymbol{r}} \mid \underline{\boldsymbol{\rho}}}^{(n)}$, (with $\left.\underline{\boldsymbol{r}} \equiv \boldsymbol{r}_{1}, \boldsymbol{r}_{2}, \cdots, \boldsymbol{r}_{n}\right)$ :

$$
C^{(n)}\left(\boldsymbol{r}_{1}, \cdots, \boldsymbol{r}_{n}, t\right)=\int d \underline{\boldsymbol{\rho}} \mathcal{P}_{\underline{\boldsymbol{r}} \mid \underline{\boldsymbol{\rho}}}^{(n)}(t) C^{(n)}\left(\boldsymbol{\rho}_{1}, \cdots, \boldsymbol{\rho}_{n}, 0\right) .
$$

In writing this equation we made explicit use of the fact that the initial distribution of the passive field $\phi(\boldsymbol{r}, 0)$ is statistically independent of the advecting velocity field. Thus the operator $\mathcal{P}_{\underline{r} \underline{\underline{\rho}} \underline{\rho}}^{(n)}$ can be written explicitly

$$
\mathcal{P}_{\underline{\boldsymbol{r}} \underline{\underline{\rho}}}^{(n)}(t) \equiv\left\langle\boldsymbol{R}\left(\boldsymbol{r}_{1}, \boldsymbol{\rho}_{1}, t\right) \boldsymbol{R}\left(\boldsymbol{r}_{2}, \boldsymbol{\rho}_{2}, t\right) \cdots \boldsymbol{R}\left(\boldsymbol{r}_{n}, \boldsymbol{\rho}_{n}, t\right)\right\rangle .
$$

The key finding [2, 3] is that the operator $\mathcal{P}_{\underline{r} \underline{\underline{\rho}}}^{(n)}$ possesses left eigenfunctions of eigenvalue 1, i.e. there exist functions $Z^{(n)}\left(\boldsymbol{r}_{1}, \boldsymbol{r}_{2} \cdots \boldsymbol{r}_{n}\right)$ satisfying

$$
Z^{(n)}\left(\boldsymbol{r}_{1}, \cdots, \boldsymbol{r}_{n}\right)=\int d \underline{\boldsymbol{\rho}} \underline{\mathcal{P}}_{\underline{\boldsymbol{r}} \underline{\mid}}^{(n)}(t) Z^{(n)}\left(\boldsymbol{\rho}_{1}, \cdots, \boldsymbol{\rho}_{n}\right) .
$$


The functions $Z^{(n)}$ are referred to as "statistically preserved structures", being invariant to the dynamics, even though the operator is strongly time dependent and decaying. How to form from these functions infinitely many conserved variables in the decaying problem was shown in [2]. The functions $Z^{(n)}(\underline{\boldsymbol{r}})$ are homogeneous functions of their arguments, with anomalous scaling exponents $\zeta_{n}$. More importantly, it was shown that the correlation functions of the forced case, $F^{(n)}(\underline{\boldsymbol{r}})$, have exactly the same scaling exponents as $Z^{(n)}(\underline{\boldsymbol{r}})[\overline{3}$. In the scaling sense

$$
F^{(n)}(\underline{\boldsymbol{r}}) \sim Z^{(n)}(\underline{\boldsymbol{r}}) .
$$

This is how anomalous scaling in passive fields is understood.

Consider next the dynamics of an active field. For concreteness we will think about temperature in turbulent Boussinesq convection, but the ideas are immediately generalized to any other type of active field. The equation of motion is formally identical to Eq. (11):

$$
\frac{\partial T(\boldsymbol{r}, t)}{\partial t}+\boldsymbol{u}(\boldsymbol{r}, t) \cdot \boldsymbol{\nabla} T(\boldsymbol{r}, t)=\kappa \nabla^{2} T(\boldsymbol{r}, t)+f(\boldsymbol{r}, t),
$$

but now the velocity field is affected by the temperature. For an incompressible fluid of unit density [6],

$$
\frac{\partial \boldsymbol{u}}{\partial t}+\boldsymbol{u} \cdot \boldsymbol{\nabla} \boldsymbol{u}=-\nabla p+\nu \nabla^{2} \boldsymbol{u}+\alpha g T \hat{z} .
$$

Here $p, \nu, \alpha, g$ and $\hat{z}$ are the pressure, kinematic viscosity, thermal expansion coefficient, acceleration due to gravity and a unit vector in the upward direction respectively. The appearance of $T$ in the equation for $\boldsymbol{u}$ is crucial, and changes the scaling exponents of $\boldsymbol{u}$ from Kolmogorov (approximately) to Bolgiano (approximately) [6]. It makes no sense now to consider the decaying problem for $T$; as this field decays, the statistics of the velocity field changes, and there is very little to say. On the other hand, we can learn a great deal from considering the forced solutions. We note that both the passive and the active forced equations can be solved in the same way:

$$
\begin{aligned}
T(\boldsymbol{r}, t) & =\int d \boldsymbol{r}^{\prime} \boldsymbol{R}\left(\boldsymbol{r}, \boldsymbol{r}^{\prime}, t\right) T\left(\boldsymbol{r}^{\prime}, 0\right) \\
& +\int d \boldsymbol{r}^{\prime} \int_{0}^{t} d \tau \boldsymbol{R}\left(\boldsymbol{r}, \boldsymbol{r}^{\prime}, t-\tau\right) f\left(\boldsymbol{r}^{\prime}, \tau\right) .
\end{aligned}
$$

with a similar expression for the passive scalar. For $t \rightarrow$ $\infty$, the first term decays to zero, but the second term exists. Next we encounter the difference between the active and passive case. Computing the average of $T$, or any other odd moment, we get a finite result:

$$
\langle T(\boldsymbol{r}, t)\rangle=\int d \boldsymbol{r}^{\prime} \int_{0}^{t} d \tau\left\langle\boldsymbol{R}\left(\boldsymbol{r}, \boldsymbol{r}^{\prime}, t-\tau\right) f\left(\boldsymbol{r}^{\prime}, \tau\right)\right\rangle .
$$

We cannot decorrelate the random forcing $f$ from the operator $\boldsymbol{R}$ which involves the velocity field. The forcing $f$ is correlated with $T$, which is itself correlated, via
Eq.(11), with $\boldsymbol{u}$, and therefore the field $T$ has a first and higher odd moments: the probability density function (pdf) of $T$ will be asymmetric. For the passive case, the equivalent Eq. (13) can be simplified by decorrelating $f$ from $\boldsymbol{R}$. As the odd moments of $f$ vanish, the passive scalar has zero odd moments, and its pdf is symmetric. This difference is fundamental.

We now argue that all the even moments, and all even correlation functions of the active and passive fields may be identical to leading scaling order. Consider for example the second order correlation function $F^{(2)}\left(\boldsymbol{r}_{1}, \boldsymbol{r}_{2}\right)$ of the active scalar:

$$
\begin{aligned}
& F^{(2)}\left(\boldsymbol{r}_{1}, \boldsymbol{r}_{2}\right)=\int d \boldsymbol{r}^{\prime} d \boldsymbol{r}^{\prime \prime} \int_{0}^{t} d \tau^{\prime} d \tau^{\prime \prime} \\
& \left\langle\boldsymbol{R}\left(\boldsymbol{r}, \boldsymbol{r}^{\prime}, t-\tau^{\prime}\right) f\left(\boldsymbol{r}^{\prime}, \tau^{\prime}\right) \boldsymbol{R}\left(\boldsymbol{r}, \boldsymbol{r}^{\prime \prime}, t-\tau^{\prime \prime}\right) f\left(\boldsymbol{r}^{\prime \prime}, \tau^{\prime \prime}\right)(14)\right.
\end{aligned}
$$

As before, in the case of the active scalar the forces cannot be decorrelated from the operators. For the passive scalar this can be done with impunity, leading to the correct form of the correlation function, i.e.

$$
\begin{aligned}
\left\langle\phi\left(\boldsymbol{r}_{1}\right) \phi\left(\boldsymbol{r}_{2}\right)\right\rangle_{f} & =\int d \boldsymbol{\rho}_{1} d \boldsymbol{\rho}_{2} \int_{0}^{t} d \tau \mathcal{P}_{\boldsymbol{r}_{1}, \boldsymbol{r}_{2} \mid \boldsymbol{\rho}_{1} \boldsymbol{\rho}_{2}}^{(2)}(t-\tau) \\
& \times\left\langle f\left(\boldsymbol{\rho}_{1}\right) f\left(\boldsymbol{\rho}_{2}\right)\right\rangle .
\end{aligned}
$$

It was shown in [3] how this expression indeed yields the forced correlation function in agreement with Eq. (9). We observe however that any correlation function $\langle A B\rangle$ can be written in terms of its Gaussian decomposition and the "connected part": $\langle A B\rangle=\langle A\rangle\langle B\rangle+\langle\tilde{A} \tilde{B}\rangle$, where $\tilde{A} \equiv A-\langle A\rangle$. Identify now $A$ with the product of operators $\boldsymbol{R}$ in Eq. (14), and $B$ with the product of forcing, and realize that Eq.(15) is the Gaussian decomposition contribution to Eq. (14). So the solution (15) exists as one contribution to (14), the other being what remains of the correlation, i.e. the part in which the forcing cannot be decorrelated from the operator. A similar phenomenon occurs for all the even order correlation functions. But we know from the theory of the passive scalar that the solution (15) and the equivalent ones for all the even-order correlation functions are identified with statistically preserved structures whose scaling is anomalous [3]. We propose that the contributions to the even-order correlation of the active scalar which are identical to the even-order correlations of the passive scalar are leading, and are therefore expected to be realized in experiments and simulations. The expectation is that the connected contributions, that are purely "forced" solutions, are either subleading or have the same exponents. This is a crucial conjecture that needs to be tested against examples. In [4] it was shown that this is correct for a particular 4 th order correlation function of the active and passive fields.

To exemplify the generality and plausibility of these ideas we examine in detail a model of active and passive scalar for which the statistical object can be computed to 
high accuracy. We consider a variant of the shell model studied in ref. [7]:

$$
\begin{aligned}
\frac{\partial T_{n}}{\partial t} & =\tilde{a} k_{n}\left(u_{n-1} T_{n-1}-h u_{n} T_{n+1}\right)+\tilde{b} k_{n}\left(u_{n} T_{n-1}-h u_{n+1} T_{n+1}\right)-\kappa k_{n}^{2} T_{n}+f \delta_{n, 0} \\
\frac{\partial u_{n}}{\partial t} & =a k_{n}\left(u_{n-1}^{2}-h u_{n} u_{n+1}\right)+b k_{n}\left(u_{n} u_{n-1}-h u_{n+1}^{2}\right)-\nu k_{n}^{2} u_{n}+T_{n} \\
\frac{\partial C_{n}}{\partial t} & =\tilde{a} k_{n}\left(u_{n-1} C_{n-1}-h u_{n} C_{n+1}\right)+\tilde{b} k_{n}\left(u_{n} C_{n-1}-h u_{n+1} C_{n+1}\right)-\kappa k_{n}^{2} C_{n}+f \delta_{n, 0} .
\end{aligned}
$$

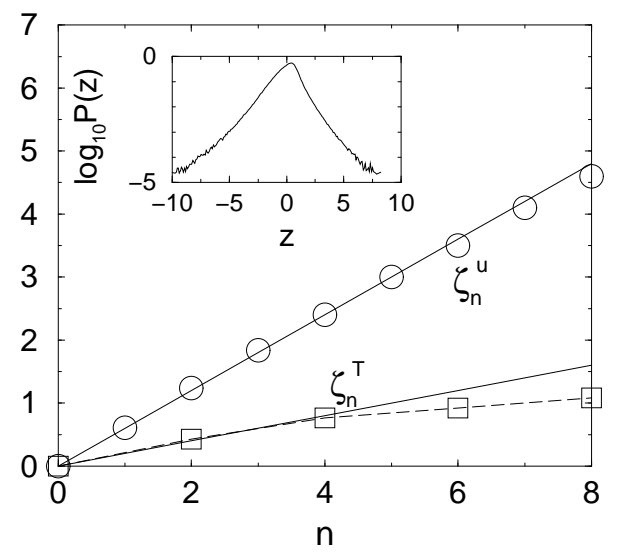

FIG. 1: The scaling exponents for the velocity field (circles) and the active scalar field (squares). The solid lines are respectively $3 n / 5$ and $n / 5$ for the velocity and the active scalar fields. Shown in the inset is the pdf of $z=\tilde{u}_{n} /\left\langle\tilde{u}_{n}^{2}\right\rangle^{1 / 2}$ at shell $n=14$.

In this model $n$ stands for index of a shell of k-vector $k_{n}=k_{0} h^{n}$, with $n=0,1, \cdots, N-1$. We take $h=2$, and the parameters used in the simulation are $a=0.01$, $\tilde{a}=\tilde{b}=b=1, k_{0}=1, \kappa=\nu=5 \times 10^{-4}$. The number of shells is $N=30$.

Without the coupling to $T_{n}$, the velocity equation has an inviscid unstable Kolmogorov fixed point, $u_{n} \sim k_{n}^{-1 / 3}$. This is changed by the coupling [7], and the system of equations for $T_{n}$ and $u_{n}$ exhibits an inviscid unstable Bolgiano fixed point, $u_{n} \sim k_{n}^{-3 / 5}, T_{n} \sim k_{n}^{-1 / 5}$. The chaotic dynamics renders the statistics of the velocity strongly non-Gaussian, cf. inset in Fig 1. The exponents for the active scalar are markedly anomalous, whereas for the velocity appear closer to normal, see Fig. 1.

To demonstrate the first point of the fundamental difference between the active and passive fields, we show in Fig. 2 the pdf's of $x=\tilde{\phi}_{n} /\left\langle\tilde{\phi}_{n}^{2}\right\rangle^{1 / 2}$ where $\tilde{\phi}$ is $\tilde{T}_{n}$ or $C_{n}$, for $n=14$. One clearly sees the symmetry of the pdf of the passive scalar, in contradistinction to the asymmetry of the pdf of the active scalar. This is typical to all $n$ in the inertial range. This is a demonstration of the discussion after Eq.(13). For the passive scalar

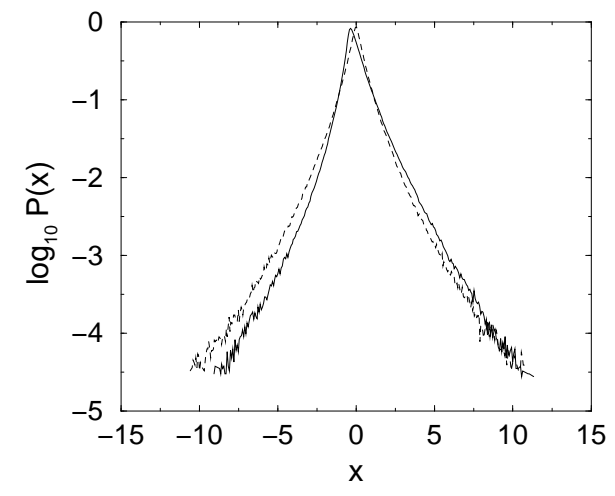

FIG. 2: The pdf's of the active (solid) and passive (dashed) scalars at shell $n=14$. Note that the pdf of the active scalar is asymmetric.

the odd moments vanish, whereas for the active scalar they all exist. The situation is altogether different for the statistics of even moments. To demonstrate the difference we plot in Fig. 3 the (typical) pdf of $\tilde{T}_{n}^{2}$ and $C_{n}^{2}$ for $n=9$ and 14 . In plotting we realize that the passive scalar is defined up to a constant, so for the passive scalar the pdf is plotted for the rescaled variable $\beta C_{n}^{2}$, where $\beta=\left\langle\tilde{T}_{n}^{2}\right\rangle /\left\langle C_{n}^{2}\right\rangle \approx 0.6327$. Note that there is only one numerical freedom $\beta$, constant for all $n$ in the inertial range. We find very close agreement of all the pdf's in the inertial range. The identity of the pdf's of $\tilde{T}_{n}^{2}$ and $C_{n}^{2}$ translates automatically to the identity (up to a constant $\beta^{m}$ ) of the even-order structure functions $S^{(2 m)}\left(k_{n}\right) \equiv\left\langle\tilde{\phi}_{n}^{2 m}\right\rangle$, where $\tilde{\phi}_{n}=\tilde{T}_{n}$ or $C_{n}$. This is demonstrated in Fig. 4. We see that the 2nd, 4th and 6th-order structure functions are barely distinguishable, with the same scaling exponents in the inertial range. Finally, the identity of the statistics of the squares of the passive and active scalars transcends structure functions. We consider next multi-point correlation functions, and in Fig. 国 compare the correlation functions $\left\langle\tilde{T}_{n}^{2} \tilde{T}_{n+5}^{2}\right\rangle$ and $\left\langle\tilde{T}_{n}^{2} \tilde{T}_{n+5}^{2} \tilde{T}_{2 n}^{2}\right\rangle$ to their passive counterparts. The conclusion is that again the multi-point correlation functions are indistinguishable once the passive ones are rescaled by $\beta^{q}$ where $q$ is the over-all order of the correlation func- 


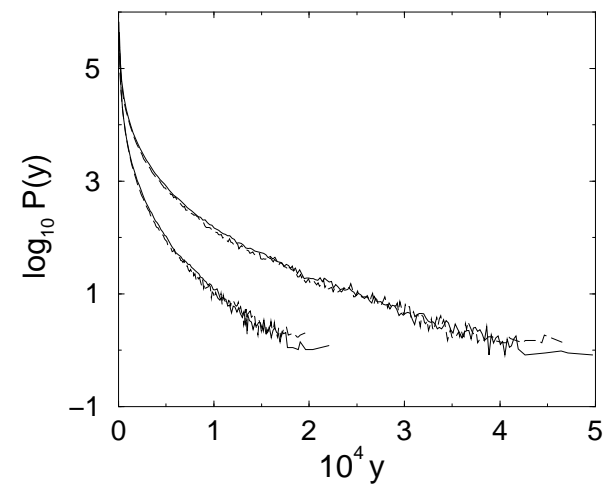

FIG. 3: The pdf's of $y$ where $y=\tilde{T}_{n}^{2}$ (solid) or $y=\beta C_{n}^{2}$ (dashed) at shells $n=9$ and 14 .

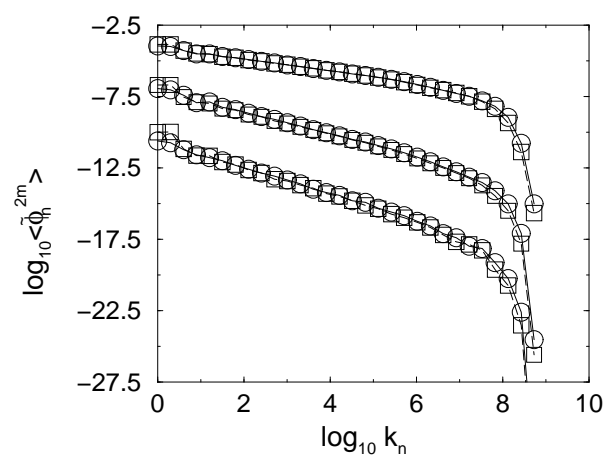

FIG. 4: The even-order structure functions $\left\langle\tilde{T}_{n}^{2 m}\right\rangle$ (circles) and $\left\langle\beta^{m} C_{n}^{2 m}\right\rangle$ (squares), with $m=1,2$ and 3 , from top to bottom.

tion. In summary, we offered a discussion and detailed numerical evidence to support the conjecture that under generic conditions the even-order correlation functions of active scalars can be understood via the emerging theory of Statistically Preserved Structures of the passive scalar counterpart. We have by now two examples, together

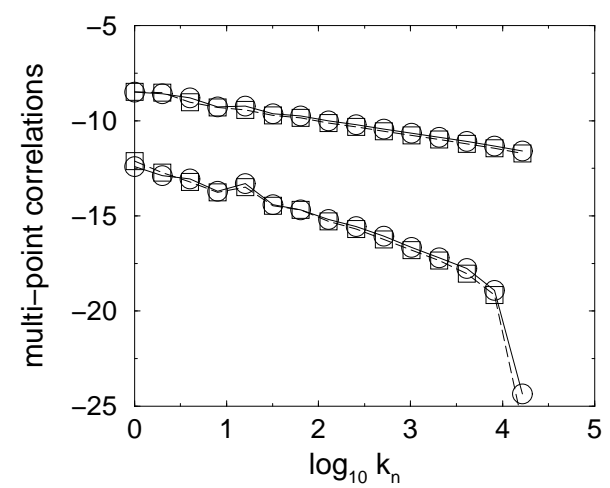

FIG. 5: Upper: $\log _{10}\left\langle\tilde{T}_{n}^{2} \tilde{T}_{n+5}^{2}\right\rangle$ (circles) and $\log _{10}\left\langle\beta^{2} C_{n}^{2} C_{n+5}^{2}\right\rangle$ (squares). Lower: $\quad \log _{10}\left\langle\tilde{T}_{n}^{2} \tilde{T}_{n+5}^{2} \tilde{T}_{2 n}^{2}\right\rangle \quad$ (circles) and $\log _{10}\left\langle\beta^{3} C_{n}^{2} C_{n+5}^{2} C_{2 n}^{2}\right\rangle$ (squares). with [4, which share however a similar coupling of the active scalar to the velocity field. The importance of this conjecture warrants considerable further work to uncover its provisos and delineate its generality.

This work had been supported in part by the Research Grants Council of Hong Kong SAR, China (CUHK 4119/98P and CUHK 4286/00P), the European Commission under a TMR grant, the German Israeli Foundation, and the Naftali and Anna Backenroth-Bronicki Fund for Research in Chaos and Complexity. TG thanks the Israeli Council for Higher Education and the Feinberg postdoctoral Fellowships program at the WIS for financial support.

[1] A. Celani and M. Vergassola, Phys. Rev. Lett. 86, 424 (2001).

[2] I. Arad, L. Biferale, A. Celani, I. Procaccia and M. Vergassola, Phys. Rev. Lett. 87, 164502 (2001).

[3] Y. Cohen, T. Gilbert and I. Procaccia, Phys. Rev. E, in press, arXiv: nlin.CD/0107016.

[4] A. Celani, T. Matsumoto, A. Mazzino and M. Vergassola, arXiv:nlin.CD/0110016 1

[5] G. Falkovich, K. Gawedzki and M. Vergassola, Rev. Mod. Phys. 73, 2001.

[6] A.S. Monin and A.M. Yaglom, Statistical Fluid Mechanics, (MIT, Cambridge 1971).

[7] A. Brandenburg, Phys. Rev. Lett., 69, 605 (1992). 